$3-2020$

\title{
Oxidative stress and male infertility: A cross sectional study
}

\author{
Rehana Rehman \\ Aga Khan University, rehana.rehman@aku.edu \\ Sofia Amjad \\ Ziauudin University, Karachi, Pakistan \\ Hemaila Tariq \\ Aga Khan University, hemaila.tariq@scholar.aku.edu \\ Nida Zahid \\ Aga Khan University, nida.zahid@aku.edu \\ Masnoon Akhter \\ Aga Khan University, masnoon.akhter@aku.edu
}

See next page for additional authors

Follow this and additional works at: https://ecommons.aku.edu/pakistan_fhs_mc_bbs

Part of the Life Sciences Commons, and the Surgery Commons

\section{Recommended Citation}

Rehman, R., Amjad, S., Tariq, H., Zahid, N., Akhter, M., Ashraf, M. (2020). Oxidative stress and male infertility: A cross sectional study. JPMA. The Journal of the Pakistan Medical Association, 70(3), 461-466.

Available at: https://ecommons.aku.edu/pakistan_fhs_mc_bbs/801 


\section{Authors}

Rehana Rehman, Sofia Amjad, Hemaila Tariq, Nida Zahid, Masnoon Akhter, and Mussarat Ashraf 


\title{
Oxidative stress and male infertility: a cross sectional study
}

Rehana Rehman1, Sofia Amjad², Hemaila Tariq³, Nida Zahid4', Masnoon Akhter5, Mussarat Ashraf6

\begin{abstract}
Objective: To compare stress markers and antioxidants in fertile and infertile males, and to explore their effects on reproductive hormones and fertility.

Methods: The cross-sectional case-control study was conducted from July 2017 to July 2018 at the Islamabad Clinic Serving Infertile Couples, Islamabad, Pakistan, and comprised male subjects aged 2555 years. Infertile subjects were the cases, while healthy fertile males acted as the controls. Stress hormones cortisol and adrenaline and antioxidants glutathione peroxidase and superoxide dismutase were measured using enzyme-linked immunosorbent assay. Data was analysed using SPSS 22.

Results: Of the 376 subjects, 241(64\%) were cases and 135(36\%) were controls. Median cortisol, adrenaline, superoxide dismutase and glutathione levels were significantly higher among the cases compared to te controls $(p<0.05)$. Follicle stimulating hormone and luteinizing hormone levels were higher in cases compared to the controls $(p=0.05)$. Mean testosterone level was higher among the controls than the cases $(p<0.001)$. After adjusting for other covariates, every increase of 7 units in cortisol increased the prevalence of infertility by $3 \%(p=0.001)$. There was significant interaction between luteinizing hormone and testosterone in the final model $(p<0.05)$.

Conclusion: Stress together with decrease in antioxidants was found to play a significant role in reducing the fertilising potential of male infertile subjects.
\end{abstract}

Keywords: Oxidative stress, Male infertility, Cortisol, Glutathione peroxidase.

(JPMA 70: 461; 2020). https://doi.org/10.5455/JPMA.12992

\section{Introduction}

Male infertility is reported to affect $5-7 \%$ of the general male population and it accounts for $20 \%$ of the cause of infertility and $50 \%$ cases of male infertility are idiopathic in nature. ${ }^{1}$ It has been reported that oxidative stress (OS) is one of the important causes of male infertility (30-80\%) as it has adverse effects on both the structural and functional integrity of the sperm, and causes loss of sperm function. 2 Spermatozoa have antioxidant defence mechanisms, to reduce reactive oxygen species (ROS) and protect gonadal cells and mature spermatozoa from oxidative damage, but this defence system has limited capacity. (2) OS is produced if the uncontrolled production of ROS exceeds the total antioxidant capacity (TAC) of the seminal plasma. ${ }^{3}$ Both fluidity and permeability of the sperm plasma membrane as well as integrity of

1,5,6Department of Biological and Biomedical Sciences, Aga Khan University, Karachi, Pakistan; 2 Department of Physiology, Ziauudin University, Karachi, Pakistan; 33rd Year MBBS Student, Aga Khan University, Karachi, Pakistan; ${ }^{4}$ Department of Surgery, Aga Khan University Hospital, Karachi, Pakistan.

Correspondence: Mussarat Ashraf. e-mail: mussarat.ashraf@aku.edu deoxyribonucleic acid (DNA) in the sperm nucleus are affected by OS, thus causing apoptosis and increased risk of male infertility. ${ }^{4}$ Unfortunately, spermatozoa, due to lack of the necessary cytoplasmic-enzyme repair systems, are not capable to restore the OS-induced damage. 5 OS can cause a number of clinical manifestations which include reduced fertilisation rates, failure of implantation, impaired embryonic development, recurrent pregnancy loss and poor assisted reproductive technology (ART) outcomes. 6

Stress and infertility are closely related to each other. ${ }^{7}$ Long-term stress produces elevated cortisol levels which is found to be associated with male infertility owing to decreased conversion of androstenedione to testosterone, thereby reducing sperm volume and concentration. ${ }^{8}$ Glucocorticoids have both central and peripheral effects on reproductive function. ${ }^{9}$ Glucocorticoids affect the hypothalamic pituitary axis (HPA) by exerting suppressive effects on the release of gonadotrophin Releasing Hormone $(\mathrm{GnRH})$ at the level of the hypothalamus, the release of luteinizing hormone $(\mathrm{LH})$ and follicle stimulating

Vol. 70, No. 3, March 2020 
hormone (FSH) at the level of anterior pituitary gland and on the testes. ${ }^{9}$ The direct effect of glucocorticoids causes inhibition of steroid hormone production by repressing the expression of steroidogenic enzymes, reduction of the testicular response to gonadotropins, or glucocorticoid-induced apoptosis of Leydig and germ cells. Glucocorticoids may also have control of Leydig cell number through arrest of cell cycle. ${ }^{9}$ Short-term stress affects male fertility by releasing catecholamines. Adrenaline is reported to decrease testosterone levels in the body by inhibiting testosterone production at HPA as well as by blocking retrovirus-associated DNA sequences

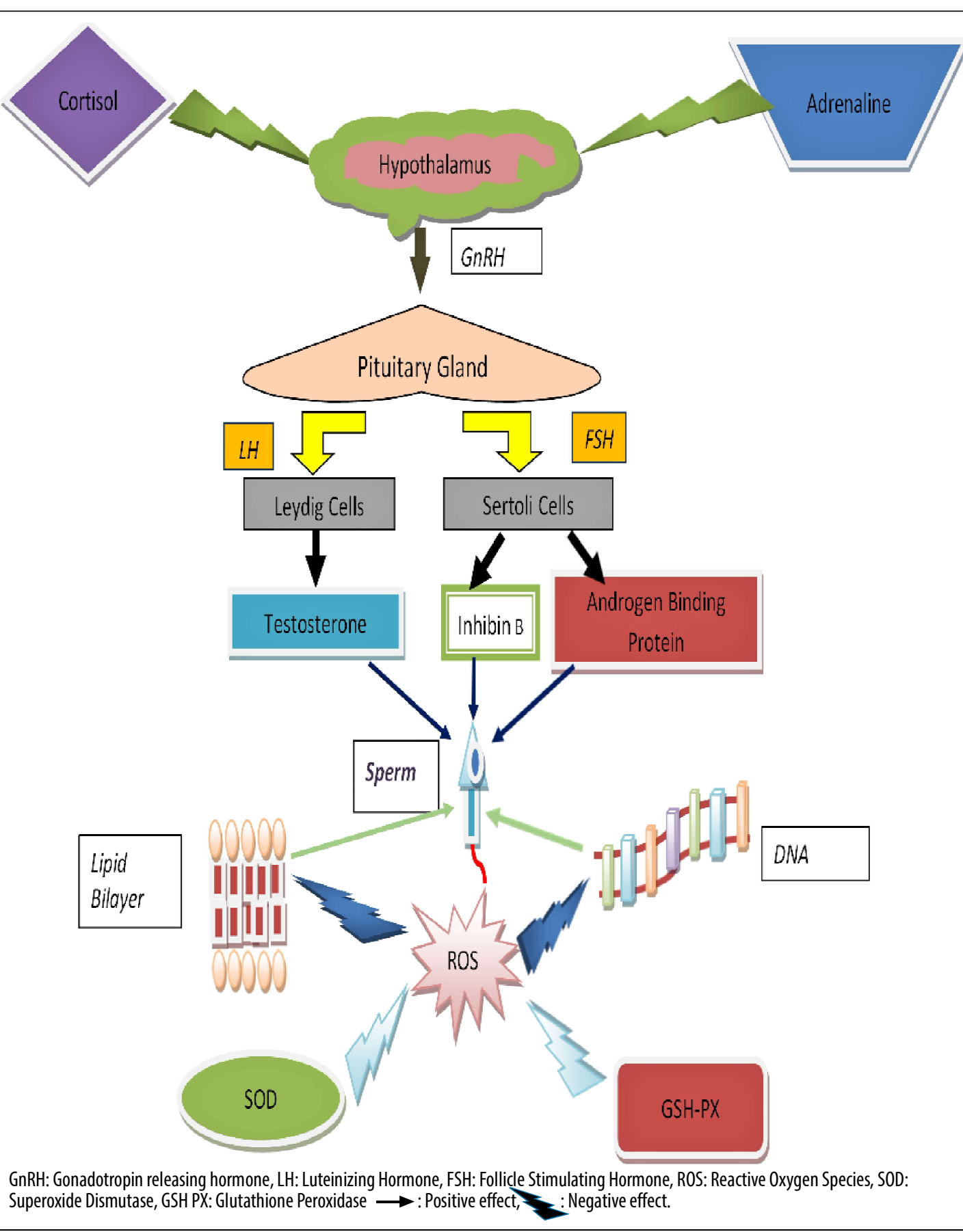

Figure: Impact of stress markers on male infertility
(RAS) pathway. ${ }^{10}$

Superoxide dismutase (SOD) is also an important marker of male infertility as studies have shown the positive association between SOD and sperm quality in terms of sperm motility a n d concentration. ${ }^{11}$ SOD, an antioxidising enzyme normally produced and secreted in seminal plasma, is found to be high in Sertoli cells and germ cells to maintain spermatogenesis in developing and maturing spermatozoa. ${ }^{12}$ This enzyme protects sperm from OS, lipid peroxidation, DNA damage, especially mitochondrial DNA, which leads to decreased adenosine triphosphate (ATP) production and energy availability, impeding forward movement of sperm and thus affecting sperm motility. ${ }^{12}$ Hence, low SOD activity is associated with infertility.

Another antioxidant enzyme, glutathione peroxidase (GPX), essential for normal sperm function due to its defensive action against 
ROS, is considered to be a useful marker for male infertility. 12 All the three isoforms of GPX, which are cytosolic, mitochondrial and nuclear, are considered to be important for spermatogenesis. The cytosolic isoform is essential for embryonic spermatogenesis whereas the mitochondrial isoform GPX is essential for sperm quality, motility and mitochondrial membrane potential. ${ }^{13}$

The current study was planned to compare the stress markers and antioxidants in fertile and infertile males and to explore their effects on male infertility. The hypothesis was that stress, as estimated by increase in adrenaline and cortisol, with impairment of antioxidants, disturbs $\mathrm{FSH}, \mathrm{LH}$ and testosterone levels required for normal reproductive functions (Figure).

\section{Patients and Method}

The cross-sectional case-control study was conducted from July 2017 to July 2018 at the Islamabad Clinic Serving Infertile Couples (ICSI), Islamabad, Pakistan. After acquiring approval from the institutional ethics review committee, the sample size was calculated using Open Source Epidemiologic Statistics for Public Health ${ }^{14}$ to compare the mean cortisol, GPX, SOD and adrenaline levels in fertile and infertile men adjusting for $10 \%$ non-response rate. Level of significance was set at $5 \%$, power for detecting the true effect $80 \%$ and anticipated difference in mean levels of the stress markers at -0.27 .15 Against the required sample size of 398, the final sample had 22(5.5\%) fewer individuals on account of a number of refusals to provide semen samples.

Those included were male subjects aged $25-55$ years. The control group comprised healthy fertile males without any history of fertility problems and whose spouses conceived within one year of unprotected intercourse. ${ }^{16}$ The cases comprised male subjects referred to the infertility clinic and were from couples who had failed to conceive after one year of regular unprotected intercourse. Those excluded were individuals who had female cause of infertility, suffered from secondary infertility, had cryptorchidism, testicular trauma, orchitis or testicular hypotrophy. Subjects having diabetes, hypertension, arthritis, malignancy, epilepsy, tuberculosis, endocrinal disorders, liver/renal diseases, and those on drugs and had conditions known to influence OS and serum cortisol level were also excluded and so were those receiving testosterone or thyroxin replacement therapy or steroids. After taking consent from each subject, general physical examination, assessment of height and weight. The calculation of body mass index (BMI) and estimation of body fat (\%) was done by bioelectrical impedance analyzer (BIA). BIA, a safe, non-invasive and portable device was used to assess the percentage of body fat composition of the participants from the estimate of entire body water. Foot-to-foot (FF) BIA scales is more acceptable and convenient as the subject only needs to stand without shoes on the scale for simultaneous measurements of BW (body weight) and impedance across the lower limbs. By adding the gender, age and height manually into the system, the fat mass (FM) of the subject or percentage fat mass (\% FM) is displayed immediately. ${ }^{17}$ The BMI of the study participants was measured and categorized according to the Asian criteria, the normal weight (18-22.9 $\left.\mathrm{kg} / \mathrm{m}^{2}\right)$, overweight $\left(23-24.9 \mathrm{~kg} / \mathrm{m}^{2}\right)$ and obesity $(\geq 25$ $\left.\mathrm{kg} / \mathrm{m}^{2}\right)$ (WHO, 2004). The cut-offs used for BF\% were normal weight (12\%-22\%), overweight (22.1\%-27\%) and obese $(>27.1 \%) .{ }^{18}$ Blood samples were collected from the subjects at 9 am and the serum from each was separated, centrifuged and analysed. Cortisol was analysed using the cortisol enzyme-linked immunosorbent assay (ELISA) kit (catalogue number: CO103S, CALBIOTECH) with a detection range of $50-230 \mathrm{ng} / \mathrm{mL}$ from 8am to $11 \mathrm{am}$ and $30-150 \mathrm{ng} / \mathrm{mL}$ at $4 \mathrm{pm}$. Adrenaline was analysed using Human Epinephrine/Adrenaline (EPI) ELISA kit (catalogue number: 95362, Glory Science Co., Ltd), with a detection range of $5 \mathrm{ng} / \mathrm{L}-1000 \mathrm{ng} / \mathrm{L}$. SOD was analysed using Human SOD ELISA kit (catalogue number: 11086, Glory Science Co., Ltd) according to the the manufacturer's protocol. GPX was analysed using Human GPX ELISA kit (catalogue number: 10800, Glory Science Co., Ltd), with a detection range of 30-700 U/L.

Data was analysed using SPSS 22. Descriptive statistics were computed for categorical variables by computing their frequencies and percentages, assessed by chi-square/ Fishers exact test. The distribution of quantitative variables was computed by their means and standard deviation (SD) / median and interquartile range (IQR) depending on the normality of data and assessed by independent $t$ test / Mann Whitney test. Unadjusted and adjusted prevalence ratios (PRs) with their $95 \%$ confidence intervals (Cls) were calculated using simple and multiple Cox Proportional hazard algorithm. Statistical significance was set at $\mathrm{p}<0.05$.

Vol. 70, No. 3, March 2020 
Table-1: Demographic, Biochemical characteristics of infertile and fertile males.

\begin{tabular}{|c|c|c|c|}
\hline Factors & Infertile $(n=241)$ & Fertile $(n=135)$ & $p$-value \\
\hline Age (in years) Mean \pm SD & $33.90 \pm 5.97$ & $37.65 \pm 6.13$ & $<0.001^{*}$ \\
\hline BMI (in kg/m²) (Mean \pm SD) & $26.77 \pm 3.58$ & $24.76 \pm 3.085$ & $<0.001^{*}$ \\
\hline BMI (in) & & & $<0.001^{*}$ \\
\hline$\leq 24 \mathrm{~kg} / \mathrm{m}^{2}$ & $56(23.2 \%)$ & $57(42.2 \%)$ & \\
\hline$>24 \mathrm{~kg} / \mathrm{m}^{2}$ & $185(76.8 \%)$ & $78(57.8 \%)$ & \\
\hline Body fat $\%($ Mean \pm SD ) & $34.53 \pm 4.67$ & $32.97 \pm 4.06$ & $<0.01^{*}$ \\
\hline Body fat $\%$ & & & 0.32 \\
\hline$<25 \%$ & $3(1.2 \%)$ & $4(3.0 \%)$ & \\
\hline $25-29.99 \%$ & $39(16.2 \%)$ & $26(19.3 \%)$ & \\
\hline$>29.99 \%$ & $199(82.6 \%)$ & $105(77.8 \%)$ & \\
\hline Smoking status & & & $0.002^{*}$ \\
\hline Smoker & $187(77.6 \%)$ & $85(63.0 \%)$ & \\
\hline Non smoker & $54(22.4 \%)$ & $50(37.0 \%)$ & \\
\hline SHBG (nmol/L) Median (IQR) & $16.40(13-16.39)$ & $30.71(25.6-41.7)$ & $<0.001^{*} \wedge$ \\
\hline Cortisol ng/mL Median (IQR) & $168.16(122.3-212.3)$ & $82.89(77.5-123.3)$ & $<0.001^{*} \wedge$ \\
\hline \multicolumn{4}{|c|}{ Adrenaline (ng/L) } \\
\hline Median (IQR) & $33.72(20-49.67)$ & $19.53(11-36.6)$ & $0.03 * \wedge$ \\
\hline SOD (ng/L) Median (IQR) & $22.36(10.2-37.14)$ & $10.80(0.5-42.7)$ & 0.02 \\
\hline \multicolumn{4}{|c|}{ Glutathione peroxidase (U/L) } \\
\hline Median (IQR) & $60.66(44.5-114.52)$ & $73.05(42.8-160.7)$ & $0.005^{*} \wedge$ \\
\hline FSH (mlU/ml) Mean \pm SD & $9.61 \pm 1.39$ & $9.30 \pm 1.31$ & $10.03^{*}$ \\
\hline LH (mlU/ml) Mean \pm SD & $10.91 \pm 5.98$ & $6.97 \pm 1.81$ & $<0.001^{*}$ \\
\hline Testosterone $(\mathrm{ng} / \mathrm{ml})$ Mean \pm & \pm SD $3.54 \pm 1.57$ & $6.05 \pm 0.82$ & $<0.001^{*}$ \\
\hline \multicolumn{4}{|c|}{$\begin{array}{l}\text { * Significant at p value }<0.05 \text { (Independent t test/ Mann Whitney test was used), } \\
\text { * } \wedge \text { Significant at p value }<0.05 \text { (Chi-square test of independence/ Fishers Exact test was } \\
\text { used), SD: Standard deviation; BMI: Body mass index; SHBG: Sex hormone-binding } \\
\text { globulin' IQR: Interquartile range; SOD: Superoxide dismutase; FSH: Follicle stimulating } \\
\text { hormone; LH: Luteinizing hormone. }\end{array}$} \\
\hline
\end{tabular}

\section{Results}

Of the 376 subjects, $241(64 \%)$ were cases and 135(36\%) were controls. The mean age of the cases was significantly lower compared to the controls $(p<0.001)$. The mean BMI and body fat (\%) were significantly higher among the cases compared to the controls $(p<0.05)$.

The median sex hormone-binding globulin (SHBG) and GPX were significantly higher in controls $(p<0.05)$. However, median cortisol, adrenaline levels and SOD were significantly higher in the cases $(p<0.05)$.

Mean FSH and LH levels were higher in cases $(p<0.05)$, and mean testosterone was higher in controls $(p<0.001)$. There was a significant weak positive correlation between cortisol and FSH ( $r=0.110, p=0.03)$, and between cortisol and $\mathrm{LH}(\mathrm{r}=0.342, \mathrm{p}<0.001)$. However, there was a significant moderate negative correlation between cortisol and testosterone $(r=-0.472, p<0.001)$. A significant weak positive correlation was evident between adrenaline and LH ( $r=0.147, p=0.004)$, a significant weak negative correlation between adrenaline and testosterone
Table-2: Univariate and Multivariable Analysis for factors associated with male infertility.

\begin{tabular}{|c|c|c|}
\hline Factors & $\begin{array}{l}\text { Unadjusted Prevalence } \\
\text { Ratio }(95 \% \mathrm{Cl})\end{array}$ & $\begin{array}{c}\text { Adjusted Prevalence } \\
\text { Ratio }(95 \% \text { Cl) }\end{array}$ \\
\hline Cortisol ng/mL & $1.005(1.003-1.007) *$ & $1.021(1.008-1.034)^{* *}$ \\
\hline Luteinizing Hormone (mlU/ml) & $1.044(1.023-1.065)^{* *}$ & $0.796(0.650-0.97)^{*}$ \\
\hline Testosterone (ng/ml) & $0.78(0.938-0.967)^{* *}$ & $0.478(0.380-0.602)^{*}$ \\
\hline LH*Testosterone & & $0.970(0.590-0.990)$ \\
\hline Adrenaline(ng/L) & $1.001(0.999-1.003)$ & \\
\hline Superoxide Dismutase (ng/L) & $0.996(0.992-0.999)^{*}$ & \\
\hline $\begin{array}{l}\text { Glutathione peroxidase (U/L) } \\
\text { Median (IQR) }\end{array}$ & $0.999(0.998-1.000)^{*}$ & \\
\hline SHBG (nmol/L) & $0.952(0.938-0.967) *$ & \\
\hline Age (in years) & $0.964(0.944-0.985) *$ & \\
\hline BMI (in kg/m2) & $1.059(1.022-1.096)^{*}$ & \\
\hline Normal weight (18- 22.9) (ref) & 1 & \\
\hline Over weight (23-24.9) & $1.419(1.053-1.914) *$ & \\
\hline Obese $(\geq 25)$ & $1.28(0.848-1.920)$ & - \\
\hline Body fat (in \%) & $1.028(1.000-1.057) *$ & \\
\hline Normal weight (12-22) & 1 & \\
\hline Over weight (22.1-27) & $1.400(0.433-4.530)$ & \\
\hline Obese $(>27)$ & $1.527(0.488-4.776)$ & - \\
\hline \multicolumn{3}{|l|}{ Smoking Status } \\
\hline Non smoker & 1 & \\
\hline Smoker & $1.324(0.978-1.792)^{*}$ & - \\
\hline Total Count (10 6 in ml) Mean \pm SD & $0.997(0.995-0.999)^{*}$ & \\
\hline Motility Median (IQR) & $0.992(0.987-0.997)^{*}$ & \\
\hline Morphology Mean \pm SD & $0.960(0.952-1.024)^{*}$ & - \\
\hline \multicolumn{3}{|c|}{ Rapid Linear Progression Median (IQR) $0.987(0.952-1.024)^{*}$} \\
\hline $\mathrm{FSH}(\mathrm{mlU} / \mathrm{ml})$ Mean \pm SD & $1.060(0.969-1.161)^{*}$ & - \\
\hline
\end{tabular}

*Significant at $p$ value $<0.25$ by cox proportional hazard algorithm, \# Significant at $p$ value $<0.05$ by cox proportional hazard algorithm after adjusting, Cl: Confidence interval; BMI: Body mass index; SHBG: Sex hormone-binding globulin; IQR: Interquartile range; FSH: Follicle stimulating hormone.

$(r=-0.173, p=0.001)$, and a significant weak positive correlation between GPX and testosterone $(r=0.139$, $\mathrm{p}<0.007$ ) (Table 1).

Univariate analysis showed that with every 1 year increase in age, the prevalence of infertility decreased by $4 \%$ ( $p$ $=0.001$ ). However, with every 1 unit increase in BMI and body fat (\%) the prevalence of infertility was increased by $5.9 \%(p=0.001)$ and $2.8 \%(p=0.054)$ respectively.

With every 1 unit increase in FSH and $\mathrm{LH}$, the prevalence of infertility increased by $6 \%(p=0.2)$ and $4.4 \%(p<0.001)$ respectively. However, with every 1 unit increase in testosterone levels and SHBG, the prevalence of infertility decreased by $20 \%(p<0.001)$ and $4.8 \%(p<0.001)$ respectively.

Among the OS markers, with every 1 unit increase in cortisol, the prevalence of infertility increased by $0.5 \%$ $(p=0.001)$, but with every 1 unit increase in SOD and GPX, 
the prevalence of infertility decreased by $0.4 \%(p=0.008)$ and $0.1 \%(p=0.003)$ respectively. The effect of adrenaline level was not significant $(p<0.25)$.

In the he final Cox regression algorithm, after adjusting for covariates $\mathrm{LH}$ and testosterone, with every increase of 7 units in cortisol, the prevalence of infertility increased by $2.1 \%(p=0.003)$. There was significant interaction between $\mathrm{LH}$ and testosterone in the final model (Table 2). Among patients with testosterone levels 0 , the prevalence of infertility for $\mathrm{LH}=4.4$ was $20 \%$ lower compared to patients with $\mathrm{LH}=0$. However, among patients with testosterone $=2.17$, the prevalence of infertility for $\mathrm{LH}=4.4$ was $3 \%$ lower compared to $\mathrm{LH}=0$ indicating that $\mathrm{LH}$ interacted with testosterone in its effect on infertility.

\section{Discussion}

The increased prevalence of infertility with exacerbation of psychological and economic burden of developing countries has motivated researchers to look for the relationship of infertility with various causative factors. ${ }^{19}$ OS is a potential contributor to the aetiology of male infertility due to the impairment of both the structural and functional integrity of spermatozoa. ${ }^{12}$ The current study, conducted to compare the stress markers and antioxidants in male fertile and infertile subjects, showed that cortisol and adrenaline levels were significantly higher in the infertile group compared to the fertile group. These results are consistent with literature. ${ }^{9}$ It has also been reported that persistent stressful conditions may lead to sustained increase in cortisol levels and if at the same time reduced GPX activity is also present, then this fails to counterbalance the disturbed microenvironment.12 Both physical and emotional stress cause activation of the neurons that secrete corticotropin-releasing hormone and ultimately result in higher plasma cortisol levels. ${ }^{18}$ Excess cortisol in turn diminishes the production of testosterone, thereby decreasing sperm volume and sperm parameters. ${ }^{21}$ Cortisol can also directly reduce testosterone production by blocking the transcription of genes that code for enzymes essential for testosterone synthesis. ${ }^{21}$ The significant finding of increased adrenaline levels in the infertile group is due to the decreased production of testosterone that causes infertility. ${ }^{10}$

The current study also showed that antioxidant GPX was significantly lower in infertile males. OS results from disequilibrium between the pro-oxidants and the antioxidant defence system in the body. The antioxidant defence system comprises scavenger system formed by enzymes such as GPX and SOD which protect the structure and function of the sperm from oxidative damage.22 GPX has a role in preserving the integrity of the sperm membrane that is rich in polyunsaturated fats, thereby preserving sperm motility from lipid peroxidation caused by ROS. 23

However, SOD was found to be significantly higher in the infertile group. This is contrary to the findings of literature. ${ }^{24}$ This might be due to increased levels of SOD in the local population and due to the low number of fertile patients. SOD is another important part of the antioxidant scavenger system which has an established role in protecting the polyunsaturated fatty acid of sperm membrane from OS and DNA fragmentation. ${ }^{12}$ ROS can cause germ cell apoptosis, thereby leading to decreased sperm count. As such, by combating ROS oxidative damage, SOD preserves sperm count, volume and motility, thereby serving as a positive indicator of male fertility. ${ }^{24}$

The current study showed significantly high adrenaline levels and low testosterone in infertile males. This negative association between adrenaline and testosterone has also been reported by other studies. ${ }^{25,26}$ This is because of the inhibitory effect of adrenaline and dopamine on testosterone production, both central through HPA, and peripheral by blocking testosterone release. 10

The current study has shown significant increase in FSH, $\mathrm{LH}$ and decrease in SHBG levels among infertile males compared to the fertile ones. These results have also been reported by other studies. ${ }^{27}$ It is due to involvement of SHBG in the regulation of Sertoli cell function and spermatogenesis.

The hypothesis of defective scavenging systems as a causative factor for male infertility needs to be further explored by estimation of TAC in seminal fluid in a diverse population of infertile subjects. By identifying the cause, targeted therapy may be helpful in treating male infertility. In terms of limitations, the current study did not take seminal plasma even though semen analysis is essential to diagnose infertility and basic seminal parameters are required to compare fertile and infertile subjects.

\section{Conclusion}

Increased stress hormones and decreased antioxidant

Vol. 70, No. 3, March 2020 
enzymes were observed in infertile groups. Stress, as indicated by increase in cortisol and adrenaline hormone secretion, together with decrease in antioxidants, was found to play a significant role in reducing the fertilising potential of male infertile subjects.

\section{Disclaimer: None. \\ Conflict of Interest: None.}

Source of Funding: Department of Biological \& Biomedical Sciences, Aga Khan University Hospital (AKUH), Karachi.

\section{References}

1. Plaseska-Karanfilska D, Noveski P, Plaseski T, Maleva I, Madjunkova S, Moneva Z. Genetic causes of male infertility. Balkan J Med Genet 2012;15(Suppl):31-4.

2. Bisht $S$, Faiq $M$, Tolahunase $M$, Dada R. Oxidative stress and male infertility. Nat Rev Urol 2017;14:470-85.

3. Sikka SC. Relative impact of oxidative stress on male reproductive function. Curr Med Chem 2001;8:851-62.

4. Chen SJ, Allam JP, Duan YG, Haidl G. Influence of reactive oxygen species on human sperm functions and fertilizing capacity including therapeutical approaches. Arch Gynecol Obstet 2013;288:191-9.

5. Saleh RA, Agarwal A. Oxidative stress and male infertility: from research bench to clinical practice. J Androl 2002;23:737-52.

6. Agarwal A, Bui AD. Oxidation-reduction potential as a new marker for oxidative stress: Correlation to male infertility. Investig Clin Urol 2017;58:385-99.

7. Alam F, Khan TA, Rehman R. Stress of infertility: Can the couple cope? J Pak Med Assoc 2018;68:679-80.

8. Ferlin A, Arredi B, Foresta C. Genetic causes of male infertility. Reprod Toxicol 2006;22:133-41.

9. Whirledge S, Cidlowski JA. Glucocorticoids and Reproduction: Traffic Control on the Road to Reproduction. Trends Endocrinol Metab 2017;28:399-415.

10. Tsai SC, Chiao YC, Lu CC, Doong ML, Chen YH, Shih HC, et al. Inhibition by amphetamine of testosterone secretion through a mechanism involving an increase of cyclic AMP production in rat testes. Br J Pharmacol 1996;118:984-8.

11. Murawski M, Saczko J, Marcinkowska A, Chwilkowska A, Grybos M, Banas T. Evaluation of superoxide dismutase activity and its impact on semen quality parameters of infertile men. Folia Histochem Cytobiol 2007;45(Suppl 1):S123-6.

12. Wagner $\mathrm{H}$, Cheng JW, Ko EY. Role of reactive oxygen species in male infertility: An updated review of literature. Arab J Urol 2017;16:35-43.
13. Schneider $M$, Forster $H$, Boersma $A$, Seiler $A$, Wehnes $H$, Sinowatz $\mathrm{F}$, et al. Mitochondrial glutathione peroxidase 4 disruption causes male infertility. FASEB J 2009;23:3233-42.

14. Dean AG, Sullivan KM, Soe MM. OpenEpi: Open Source Epidemiologic Statistics for Public Health, Version 3.01. [Online] 2013 [Cited 2017 July 15]. https://www.openepi.com/ SampleSize/SSCC.htm

15. Shukla KK, Mahdi AA, Ahmad MK, Jaiswar SP, Shankwar SN, Tiwari SC. Mucuna pruriens Reduces Stress and Improves the Quality of Semen in Infertile Men. Evid Based Complement Alternat Med 2010;7:137-44.

16. Menkveld R, Wong WY, Lombard CJ, Wetzels AM, Thomas CM, Merkus HM, et al. Semen parameters, including WHO and strict criteria morphology, in a fertile and subfertile population: an effort towards standardization of in-vivo thresholds. Hum Reprod 2001;16:1165-71.

17. Lazzer S, Boirie Y, Meyer M, Vermorel M. Evaluation of two foot-tofoot bioelectrical impedance analysers to assess body composition in overweight and obese adolescents. British Journal of Nutrition. 2003;90:987-92.

18. WHO Expert Consultation. Appropriate body-mass index for Asian populations and its implications for policy and intervention strategies. Lancet. 2004;363(9403):157-63. 9. World Health Organization. Obesity: prevention.

19. Amjad S, Baig M, Zahid N, Tariq S, Rehman R. Association between leptin, obesity, hormonal interplay and male infertility. Andrologia 2019;51:e13147.

20. Lima AP, Moura MD, Rosa e Silva AA. Prolactin and cortisol levels in women with endometriosis. Braz J Med Biol Res 2006;39:11217.

21. Whirledge S, Cidlowski JA. Glucocorticoids, stress, and fertility. Minerva Endocrinol 2010;35:109-25.

22. Naher ZU, Biswas SK, Mollah FH, Ali M, Arslan MI. Role of Glutathione in Male Infertility. Bangladesh J Med Biochem 2011;4:20-5.

23. Chaudhari AR, Das P, Singh R. Study of oxidative stress and reduced glutathione levels in seminal plasma of human subjects with different fertility potential. Biomed Res 2008;19:207-10.

24. Yan L, Liu J, Wu S, Zhang S, Ji G, Gu A. Seminal superoxide dismutase activity and its relationship with semen quality and SOD gene polymorphism. J Assist Reprod Genet 2014;31:549-54.

25. Mayerhofer A, Bartke A, Steger RW. Catecholamine effects on testicular testosterone production in the gonadally active and the gonadally regressed adult golden hamster. Biol Reprod 1989;40:75261.

26. Levin J, Lloyd CW, Lobotsky J, Friedrich EH. The effect of epinephrine on testosterone production. Acta Endocrinol (Copenh) 1967;55:184 92.

27. Zorn B, Osredkar J, Meden-Vrtovec H, Majdic G. Leptin levels in infertile male patients are correlated with inhibin $\mathrm{B}$, testosterone and SHBG but not with sperm characteristics. Int J Androl 2007;30:439-44. 